\section{Crianças COM hÉrNIA INGUINAL PODEM SER OPERADAS POR CIRURGIÃO GERAL? VISÃO DO ANDROLOGISTA}

Parabenizamos a iniciativa de Uenis Tannuri em questionar se crianças com hérnia inguinal podem ser operadas por cirurgião geral. Interessantemente, esta não é apenas uma questão que envolve o cirurgião geral ou o cirurgião pediátrico apenas. Esta questão envolve também o andrologista que se envolve com o problema quando o homem, agora já adulto, apresenta queixas de infertilidade decorrentes da cirurgia resultando em azoospermia de causa obstrutiva.

Herniorrafia inguinal prévia é a causa mais comum de obstrução iatrogênica dos casos deferentes. A incidência estimada é de $0,8 \%$ a $2 \%$ quando os pacientes são operados na infância. Além disso, 26,7\% dos pacientes inférteis com história de herniorrafia inguinal prévia realizada na infância apresentam como causa a obstrução unilateral dos vasos deferentes decorrente da herniorrafia inguinal. Infelizmente, a obstrução deferencial não é reconhecida durante a herniorrafia. Caso o paciente apresente um canal deferente permeável com produção de espermatozóides normal pelo testículo ipsilateral, uma obstrução do deferente colateral poderá nunca ser detectada. Por outro lado, caso o paciente apresente dano deferencial bilateral, ele irá invariavelmente apresentar infertilidade.

Recentemente, Pasqualotto et al. publicaram estudo envolvendo 20 procedimentos realizados em 13 homens diagnosticados com infertilidade e trauma nos vasos deferentes secundários à herniorrafia inguinal prévia. A taxa de permeabilidade foi de $65 \%$. No grupo de vasovasostomia, a taxa de permeabilidade foi de $60 \%$ (9/15) e no grupo de vasoepididimostomia foi de $80 \%$ (4/5). Entre os pacientes azoospérmicos, 13 procedimentos foram realizados. As taxas de permeabilidade foram de 42,9\% para a vasovasostomia (3/ 7) e 100\% para o procedimento de vasoepididimostomia (4/ 4). As taxas globais foram de $40 \%$. Quatro de cinco homens (80\%) que foram submetidos à vasoepididimostomia estabeleceram gravidez. Desta forma, a vasovasostomia microcirúrgica após trauma inguinal nos vasos deferentes resulta em taxas de permeabilidade razoáveis, mas uma taxa de gravidez baixa comparada à reversão de vasectomia.

Assim, independente do profissional que irá realizar uma herniorrafia inguinal em paciente pediátrico, é importante um extremo cuidado, talvez até necessidade de usar meios de magnificação, para evitar obstrução dos canais deferentes.

Fabio Firmbach Pasqualotto

Caxias do SUl - RS

\section{Referências}

I. Matsuda $H$, Yorii $Y$, Yoshida $O$. Unilateral obstruction of the vas deferens caused by childhood inguinal herniorrhaphy in male infertility patients. Fertil Steril. 1992;58(6):609-13.

2. Pasqualotto FF, Pasqualotto EB, Agarwal A, Thomas AJ Jr. Results of microsurgical anastomosis in men with seminal tract obstruction due to inguinal herniorrhaphy. Rev Hosp Clin Fac Med São Paulo. 2003;58(6):305-9.

3. Tannuri U. Crianças com hérnia inguinal podem ser operadas por cirurgião geral? Rev Assoc Med Bras 2005; 5 I (6):305. 